\title{
Optimización por enjambre de partículas en el diseño de un árbol de transmisión
}

\author{
Derlis Hernández-Lara ${ }^{1,3}$, Emmanuel Alejandro Merchán-Cruz ${ }^{1}$, \\ Ricardo Gustavo Rodríguez-Cañizo ${ }^{1}$, Edgar Alfredo Portilla-Flores ${ }^{2}$, \\ Álvaro Marcos Santiago-Miguel ${ }^{1}$ \\ ${ }^{1}$ Instituto Politécnico Nacional, Escuela Superior de Ingeniería Mecánica y Eléctrica, \\ México \\ ${ }^{2}$ Instituto Politécnico Nacional, Centro de Innovación y Desarrollo Tecnológico en Cómputo, \\ México \\ ${ }^{3}$ Tecnológico de Estudios Superiores de Ecatepec, \\ México \\ \{derlis392, marcoversal\}@hotmail.com, \\ \{eamerchan, rgrodriguez, aportilla\}@ipn.mx
}

\begin{abstract}
Resumen. En este trabajo se presenta la utilización de metaheurísticas para apoyar al diseño de sistemas mecánicos, como una propuesta de metodología de diseño robusta. Se empleó el algoritmo de optimización por enjambre de partículas, PSO (del inglés, Particle Swarm Optimization), para el diseño óptimo de un árbol de transmisión hecho de materiales compuestos, con el fin de obtener los parámetros de diseño adecuados para soportar las cargas específicas a las que estará sometido este elemento. El problema en este tipo de diseños, es encontrar el espesor adecuado de la pieza, es decir el número de láminas y sus respectivas orientaciones que la conformarán, para lo cual existen demasiadas posibles combinaciones al trabajar con laminados de materiales compuestos, la metodología propuesta busca la mejor solución posible de acuerdo con los parámetros del problema. La principal aportación de este trabajo es el uso de metaheurísticas para resolver problemas de diseño complejos y difíciles de resolver por métodos tradicionales. Los resultados experimentales conllevan a concluir que este tipo de implementaciones son de gran utilidad para resolver problemas de optimización numérica en el diseño con materiales compuestos para diversas aplicaciones en ingeniería.
\end{abstract}

Palabras clave: optimización por enjambre de partículas (PSO), diseño con materiales compuestos, árbol de transmisión.

\section{Particle Swarm Optimization Approach in the Drive Shaft Design}

Abstract. This paper presents the use of metaheuristics to support the design of mechanical systems, as a proposal of robust design methodology. The particle 


\begin{abstract}
swarm optimization algorithm (PSO) approach in the drive shaft made of composite materials, in order to obtain the optimal design parameters to support the loads specific to which this element will be subject. The problem in this type of design, is to find the adequate thickness of the piece, that is to say, the number of sheets and their respective orientation that will form it, for which there are too many possible combinations when working with laminates of composite materials, the proposed methodology look for the best possible solution according to the parameters of the problem. The main contribution of this work is the use of metaheuristics to solve design problems and complexes of resolution of traditional methods. The experimental results will lead to the conclusion that this type of implementations is very useful to solve problems of numerical optimization in the design with composite materials for diverse engineering applications.
\end{abstract}

Keywords: particle swarm optimization (PSO), design with composite materials, drive shaft.

\title{
1. Introducción
}

Uno de los principales objetivos al implementar metaheurísticas en problemas de optimización, es el de resolver situaciones complejas y buscar soluciones factibles dentro de un intervalo definido por las cotas de diseño. Para este estudio se realiza la búsqueda del número de laminados y mejor secuencia de apilamiento en el diseño de un árbol de transmisión para automóviles hecho de materiales compuestos, se usa el algoritmo de optimización por enjambre de partículas para obtener los parámetros de diseño adecuados para las cargas y esfuerzos requeridos en su trabajo específico.

En [1] mencionan que en las últimas décadas las industrias como la aeronáutica, automotriz y la de prótesis prefieren utilizar materiales compuestos en lugar de materiales tradicionales, debido a su excelente relación resistencia/peso y alta rigidez especifica. Otra ventaja de usar materiales compuestos es que la estructura se puede diseñar seleccionando la fibra y las orientaciones adecuadas para cumplir con los requerimientos específicos. Un problema clásico de diseño con materiales compuestos tiene gran cantidad de variables discretas, como el número de láminas, orientación de las mismas, espesor y tipo de material. La flexibilidad en seleccionar estas variables para cumplir con los requisitos introduce complejidad en el problema de diseño. Además, en la mayoría de los problemas de diseño, se conocen ciertas especificaciones a priori, como, espesor del laminado, opciones para orientaciones de los mismos y tipo de material. Por lo tanto, el diseño de una estructura mediante materiales compuestos se reduce para buscar orientaciones discretas de capas apropiadas y parámetros geométricos en un rango dado para lograr la resistencia y rigidez especificada.

\section{Antecedentes y trabajos previos}

En [2] utilizaron metaheurísticas para el diseño, optimización y selección de vigas mediante la metodología de diseño paramétrico, variando así las secciones transversales 
de las vigas de estudio, con el fin de optimizarlas para soportar cargas especificas sin fracturarse. Sus resultados experimentales concluyen que este tipo de implementaciones son de gran utilidad para resolver problemas de diseño en diversas aplicaciones.

En [3] formularon una metodología de diseño óptimo multiobjetivo de vigas de pared delgada PRF (Plásticas Reforzadas con Fibras). El problema de optimización se plantea de manera de considerar restricciones estructurales y geométricas preestablecidas en el diseño, proponen minimizar el peso de la estructura conjuntamente con los desplazamientos máximos producidos, para lo cual usan la heurística SA (Simulated Annealing) empleando dos esquemas de búsqueda diferentes.

En [1] utilizaron optimización por enjambre de partículas y algoritmos genéticos (AG) para el diseño de una viga hueca, con un enfoque multiobjetivo, el diseño de la viga fue hecho con materiales compuestos y aplicación para la industria aeronáutica, los resultados mostraron que PSO obtuvo mejores resultados que el AG para este tipo de diseños.

En [4] se diseñó un árbol de transmisión para vehículos automotores hecho de materiales compuestos, la metodología de diseño implicó encontrar la combinación adecuada del material para que la pieza no falle en su funcionamiento, en este trabajo se utilizó un algoritmo propuesto por el autor, los resultados obtenidos fueron satisfactorios, concluyendo que la utilización de heurísticas en el diseño con materiales compuestos es de gran utilidad.

En [5] usaron algoritmos genéticos para diseñar un árbol de transmisión, obteniendo el espesor y la secuencia de apilamiento óptima de materiales compuestos para la pieza, para este caso diseñaron con fibra de vidrio y fibra de carbono e hicieron la comparación con respecto al diseño con acero.

Respecto a los antecedentes mencionados, las desventajas son que los resultados obtenidos son muy ideales y difíciles de llevar a la práctica, sin embargo, esta investigación pretende contribuir a subsanar esta situación, mediante la implementación de restricciones que permitan obtener resultados factibles de materializar y que conlleven a utilizar esta metodología para diversos diseños con materiales compuestos en ingeniería.

\section{Metodología}

De acuerdo con la literatura, las heurísticas pueden adaptarse al diseño con materiales compuestos, ya que son métodos de optimización global y pueden utilizarse para problemas no lineales o de variables discretas. En ingeniería siempre hay que analizar detalladamente el fenómeno en cuestión, para poder determinar todas las variables involucradas al respecto, en este caso interesa el estudio y comprensión del diseño de un árbol de transmisión hecho de fibra de carbono.

Se inicia con una propuesta de análisis del sistema de tal forma que se diseñe adecuadamente, para este caso se analiza el sistema como se observa la figura 1. 


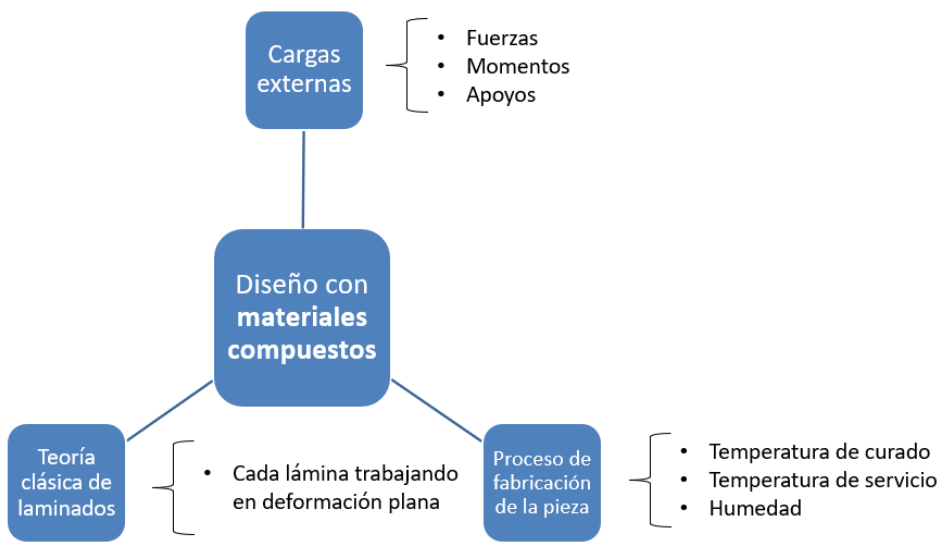

Fig. 1. Elementos a considerar en el diseño con materiales compuestos.

\subsection{Teoría clásica de laminados}

Para diseñar tomando en cuenta los esfuerzos aplicados al árbol de transmisión, y las reacciones internas que se producen en el material del cual está conformado se tiene que usar la teoría clásica de laminados. Para crear una relación lineal entre tensióndeformación para un material anisótropo se parte de la teoría de elasticidad como se muestra en la ecuación 1, mediante la ley de Hooke generalizada [4].

$$
\left\{\sigma_{i j}\right\}=\left[Q_{i j}\right]\left\{\varepsilon_{i j}\right\}, \quad \text { Siendo } i, j=1,2,3,4,5,6
$$

Donde $\left[Q_{i j}\right]$ recibe el nombre de la matriz de rigidez. Para un material genérico, esta matriz tiene 36 componentes para definir completamente el material como se muestra en la ecuación 2 .

$$
\left[Q_{i j}\right]=\left[\begin{array}{llllll}
Q_{11} & Q_{12} & Q_{13} & Q_{14} & Q_{15} & Q_{16} \\
Q_{21} & Q_{22} & Q_{23} & Q_{24} & Q_{25} & Q_{26} \\
Q_{31} & Q_{32} & Q_{33} & Q_{34} & Q_{35} & Q_{36} \\
Q_{41} & Q_{42} & Q_{43} & Q_{44} & Q_{45} & Q_{46} \\
Q_{51} & Q_{52} & Q_{53} & Q_{45} & Q_{55} & Q_{56} \\
Q_{61} & Q_{62} & Q_{63} & Q_{46} & Q_{65} & Q_{66}
\end{array}\right]
$$

Para este tipo de diseños los laminados de fibra de carbono son delgados y no se aplican cargas fuera del plano, se consideran como problemas de deformación plana, y se definen como se muestra en las ecuaciones 3 y 4 .

$$
\left\{\begin{array}{c}
\sigma_{1} \\
\sigma_{2} \\
\tau_{12}
\end{array}\right\}=[Q]\left\{\begin{array}{c}
\varepsilon_{1} \\
\varepsilon_{2} \\
\gamma_{12}
\end{array}\right\}
$$




$$
\left\{\begin{array}{c}
\sigma_{1} \\
\sigma_{2} \\
\tau_{12}
\end{array}\right\}=\left[\begin{array}{ccc}
Q_{11} & Q_{12} & 0 \\
Q_{21} & Q_{22} & 0 \\
0 & 0 & Q_{66}
\end{array}\right]\left\{\begin{array}{c}
\varepsilon_{1} \\
\varepsilon_{2} \\
\gamma_{12}
\end{array}\right\} .
$$

La matriz de rigidez para cada lámina queda definida por las ecuaciones 5 y 6 .

$$
\begin{aligned}
{\left[Q_{i j}\right] } & =\left[\begin{array}{ccc}
Q_{11} & Q_{12} & 0 \\
Q_{21} & Q_{22} & 0 \\
0 & 0 & Q_{66}
\end{array}\right], \\
{\left[Q_{i j}\right] } & =\left[\begin{array}{ccc}
\frac{E_{11}}{1-v_{12} v_{21}} & \frac{v_{12} E_{22}}{1-v_{12} v_{21}} & 0 \\
\frac{v_{12} E_{22}}{1-v_{12} v_{21}} & \frac{E_{22}}{1-v_{12} v_{21}} & 0 \\
0 & 0 & Q_{66}
\end{array}\right],
\end{aligned}
$$

donde: $E_{i j}=$ módulo de elasticidad en el eje correspondiente.

$v_{12}=$ Coeficiente de Poisson en XY.

La matriz $\left[Q_{i j}\right]$ está expresada en ejes locales. Para convertir esta matriz en ejes globales se necesita operar con la matriz de transformación como se muestra en las ecuaciones 7 y 8 .

$$
\begin{aligned}
& \left\{\sigma_{i j}^{\prime}\right\}=\left[\overline{Q_{\imath}}\right]\left\{\varepsilon_{i j}^{\prime}\right\}, \quad \text { Siendo } i, j=1,2,6 \text {. } \\
& \left\{\begin{array}{c}
\sigma_{x} \\
\sigma_{y} \\
\tau_{x y}
\end{array}\right\}=\left[\begin{array}{lll}
\overline{Q_{11}} & \overline{Q_{12}} & \overline{Q_{11}} \\
\overline{Q_{21}} & \overline{Q_{22}} & \overline{Q_{11}} \\
\overline{Q_{26}} & \overline{Q_{66}}
\end{array}\right]\left\{\begin{array}{c}
\varepsilon_{x} \\
\varepsilon_{y} \\
\gamma_{x y}
\end{array}\right\} .
\end{aligned}
$$

En las ecuaciones de la 9 a la 14 se muestra el cálculo para cada variable anterior de acuerdo con la orientación de las fibras respecto al eje local [5].

$$
\begin{aligned}
& \overline{Q_{11}}=Q_{11} c^{4}+Q_{22} s^{4}+2\left(Q_{12}+2 Q_{66}\right) c^{2} s^{2}, \\
& \overline{Q_{12}}=\left(Q_{11}+Q_{22}-4 Q_{66}\right) c^{2} s^{2}+Q_{12}\left(c^{4}+s^{4}\right), \\
& \overline{Q_{16}}=\left(Q_{11}-Q_{12}-2 Q_{66}\right) c^{3} s-\left(Q_{12}-Q_{22}+2 Q_{66}\right) c s^{3}, \\
& \overline{Q_{22}}=Q_{11} s^{4}+Q_{22} c^{4}+2\left(Q_{12}+2 Q_{66}\right) c^{2} s^{2}, \\
& \overline{Q_{26}}=\left(Q_{11}-Q_{12}-2 Q_{66}\right) s^{3} c-\left(Q_{12}-Q_{22}+2 Q_{66}\right) s c^{3}, \\
& \overline{Q_{66}}=\left(Q_{11}+Q_{22}-2 Q_{12}-2 Q_{66}\right) c^{2} s^{2}+Q_{66}\left(c^{4}+s^{4}\right)
\end{aligned}
$$

Las ecuaciones anteriores se obtuvieron tomando en cuenta el esquema mostrado en la figura 2. 


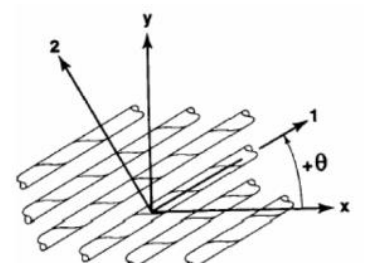

Fig. 2. En las expresiones anteriores: $\mathrm{c}=\cos \theta \mathrm{y} \mathrm{s}=\operatorname{sen} \theta$.

Según la teoría clásica de las placas laminadas, la ecuación constitutiva se puede escribir como en se muestra en la ecuación 15.

$$
\left\{\begin{array}{l}
N \\
M
\end{array}\right\}=\left[\begin{array}{ll}
A & B \\
B & D
\end{array}\right]\left\{\begin{array}{c}
\varepsilon^{0} \\
k
\end{array}\right\}
$$

donde:

$\varepsilon^{0}=$ Vector de deformaciones en el plano medio

$k=$ Curvaturas en la lámina

La forma completa de la anterior expresión se observa en la ecuación 16.

$$
\left\{\begin{array}{c}
N_{x} \\
N_{y} \\
N_{x y} \\
M_{x} \\
M_{y} \\
M_{x y}
\end{array}\right\}=\left[\begin{array}{llllll}
A_{11} & A_{12} & A_{16} & B_{11} & B_{12} & B_{16} \\
A_{21} & A_{22} & A_{26} & B_{21} & B_{22} & B_{26} \\
A_{16} & A_{26} & A_{66} & B_{16} & B_{26} & B_{66} \\
B_{11} & B_{12} & B_{16} & D_{11} & D_{12} & D_{16} \\
B_{21} & B_{22} & B_{26} & D_{21} & D_{22} & D_{26} \\
B_{16} & B_{26} & B_{66} & D_{16} & D_{26} & D_{66}
\end{array}\right]\left\{\begin{array}{c}
\varepsilon_{x}^{0} \\
\varepsilon_{y}^{0} \\
\gamma_{x y}^{0} \\
k_{x} \\
k_{y} \\
k_{x y}
\end{array}\right\} .
$$

Las direcciones de las cargas y momentos aplicados a cada lamina que constituye el árbol de transmisión se muestran en la figura 3 [6].



Fig. 3. Las fuerzas y los momentos están acoplados por la matriz $[B]$. Están definidos por unidad de longitud del lado sobre el que actúan.

Para el caso de estudio se considera un laminado simétrico, para que la pieza no se pandee después del proceso de fabricación, como se muestra en la figura 4. 


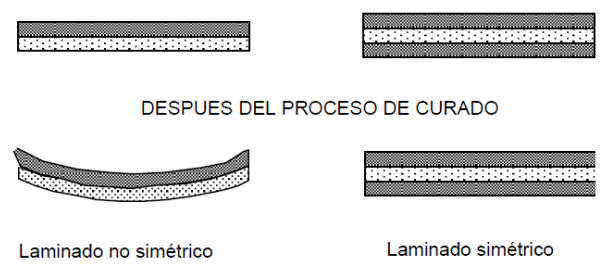

Fig. 4. Laminado simétrico.

$\mathrm{Al}$ considerar un laminado simétrico, resulta que $\left[\boldsymbol{B}_{i j}\right]=0$, por lo que se simplifica la ecuación 16 y se obtiene las ecuaciones 17 y 18 .

$$
\begin{aligned}
& \left\{\begin{array}{c}
N_{x} \\
N_{y} \\
N_{x y}
\end{array}\right\}=\left[\begin{array}{lll}
A_{11} & A_{12} & A_{16} \\
A_{21} & A_{22} & A_{26} \\
A_{16} & A_{26} & A_{66}
\end{array}\right]\left\{\begin{array}{c}
\varepsilon_{x}^{0} \\
\varepsilon_{Y}^{0} \\
\gamma_{x Y}^{0}
\end{array}\right\}, \\
& \left\{\begin{array}{c}
M_{x} \\
M_{y} \\
M_{x y}
\end{array}\right\}=\left[\begin{array}{lll}
D_{11} & D_{12} & D_{16} \\
D_{21} & D_{22} & D_{26} \\
D_{16} & D_{26} & D_{66}
\end{array}\right]\left\{\begin{array}{c}
k_{x} \\
k_{y} \\
k_{x y}
\end{array}\right\},
\end{aligned}
$$

Donde para calcular los coeficientes A, B y D, se ocupan las ecuaciones de la 19 a la 20 .

$$
\begin{aligned}
& {\left[A_{i j}\right]=\sum_{k=1}^{n}\left[\overline{Q_{l \jmath}}\right]_{k}\left(Z_{k}-Z_{k-1}\right)=\sum_{k=1}^{n}\left[\overline{Q_{l}}\right]_{k} t_{k},} \\
& {\left[B_{i j}\right]=\sum_{k=1}^{n}\left[\overline{Q_{l \jmath}}\right]_{k}\left(Z_{k}^{2}-Z_{k-1}^{2}\right),} \\
& {\left[D_{i j}\right]=\sum_{k=1}^{n}\left[\overline{Q_{l \jmath}}\right]_{k}\left(Z_{k}^{3}-Z_{k-1}^{3}\right) .}
\end{aligned}
$$

Las deformaciones en cada lamina están dadas por las ecuaciones 21 y 22 .

donde:

$$
\begin{aligned}
& \left\{\begin{array}{c}
\varepsilon_{x}^{0} \\
\varepsilon_{Y}^{0} \\
\gamma_{x Y}^{0}
\end{array}\right\}=\left[\begin{array}{lll}
a_{11} & a_{12} & a_{16} \\
a_{21} & a_{22} & a_{26} \\
a_{16} & a_{26} & a_{66}
\end{array}\right]\left\{\begin{array}{c}
N_{x} \\
N_{y} \\
N_{x y}
\end{array}\right\}, \\
& \left\{\begin{array}{c}
k_{x} \\
k_{y} \\
k_{x y}
\end{array}\right\}=\left[\begin{array}{lll}
d_{11} & d_{12} & d_{16} \\
d_{21} & d_{22} & d_{26} \\
d_{16} & d_{26} & d_{66}
\end{array}\right]\left\{\begin{array}{c}
M_{x} \\
M_{y} \\
M_{x y}
\end{array}\right\},
\end{aligned}
$$

$$
\left[\begin{array}{lll}
a_{11} & a_{12} & a_{16} \\
a_{21} & a_{22} & a_{26} \\
a_{16} & a_{26} & a_{66}
\end{array}\right]=\left[\begin{array}{lll}
A_{11} & A_{12} & A_{16} \\
A_{21} & A_{22} & A_{26} \\
A_{16} & A_{26} & A_{66}
\end{array}\right]^{-1},
$$


Derlis Hernández-Lara,Emmanuel Alejandro Merchán-Cruz, et al.

$$
\left[\begin{array}{lll}
d_{11} & d_{12} & d_{16} \\
d_{21} & d_{22} & d_{26} \\
d_{16} & d_{26} & d_{66}
\end{array}\right]=\left[\begin{array}{lll}
D_{11} & D_{12} & D_{16} \\
D_{21} & D_{22} & D_{26} \\
D_{16} & D_{26} & D_{66}
\end{array}\right]^{-1} .
$$

A partir de los cálculos anteriores, se calculan las tensiones en cada lámina en ejes globales, según las ecuaciones 25 y 26 :

$$
\left\{\begin{array}{c}
\sigma_{x} \\
\sigma_{y} \\
\tau_{x y}
\end{array}\right\}_{k}=\left[\begin{array}{lll}
\overline{Q_{11}} & \overline{Q_{12}} & \overline{Q_{11}} \\
\overline{Q_{21}} & \overline{Q_{22}} & \overline{Q_{11}} \\
\overline{Q_{16}} & \overline{Q_{26}}
\end{array}\right]_{k}\left\{\begin{array}{c}
\varepsilon_{x} \\
\varepsilon_{y} \\
\gamma_{x y}
\end{array}\right\}_{k},
$$

donde:

$$
\left\{\begin{array}{c}
\varepsilon_{x} \\
\varepsilon_{y} \\
\gamma_{x y}
\end{array}\right\}_{k}=\left\{\begin{array}{c}
\varepsilon_{x}^{0} \\
\varepsilon_{Y}^{0} \\
\gamma_{x Y}^{0}
\end{array}\right\}+Z\left\{\begin{array}{c}
k_{x} \\
k_{y} \\
k_{x y}
\end{array}\right\} .
$$

En la ecuación 26, el primer término corresponde al de deformaciones en el plano medio y el segundo al vector de curvaturas.

\subsection{Optimización por enjambre de partículas}

La optimización por enjambre de partículas (PSO) es una técnica de búsqueda desarrollada por el Dr. Eberhart y el Dr. Kennedy en 1995, inspirada en el comportamiento social de las aves, y que corresponde al tipo de inteligencia de enjambre, tiene sus raíces en la vida artificial, psicología social, ingeniería y ciencias de la computación [7].

De acuerdo con [8] PSO es un método adaptativo que utiliza agentes o partículas que se mueven a través de un espacio de búsqueda utilizando los principios de: Evaluación, Comparación e Imitación:

Evaluación: La tendencia al estímulo de evaluar, es la principal característica de los organismos vivos. El aprendizaje no ocurre a menos que el organismo pueda evaluar, pueda distinguir características del medio ambiente que atraen o características que repelen. Desde este punto de vista, el aprendizaje puede definirse como un cambio que posibilita al organismo mejorar la evaluación promedio de su medio ambiente.

Comparación: Los estándares del comportamiento social se realizan mediante la comparación con otros.

Imitación: Lorenz [8], asegura que solo los seres humanos y algunas aves son capaces de imitar. La imitación es central para la adquisición y mantenimiento de las habilidades mentales.

El proceso comienza con una población inicial aleatoria, y la búsqueda de la solución óptima se realiza según avanzan las generaciones; sin embargo, a diferencia de los algoritmos genéticos (AG), la optimización por enjambre de partículas (PSO) no necesita operadores de cruce y de mutación. Las posibles soluciones, llamadas partículas, vuelan por el espacio de búsqueda siguiendo las partículas óptimas actuales. Cada partícula realiza un seguimiento de sus coordenadas en el espacio de búsqueda, 
que se asocian con la mejor posición actual (fitness). El valor de la mejor posición actual se denomina pbest, y será almacenado en una base de datos. Cuando una partícula vecina de la posición óptima actual encuentra una posición mejor, almacena la nueva posición óptima con el nombre de lbest. A medida que se descubren nuevas y mejores posiciones, éstas pasan a orientar los movimientos de las partículas. Cuando una partícula ha recorrido todo el espacio de búsqueda, la posición óptima global se almacena con el nombre de gbest.

El método de PSO consiste en que, en cada paso de tiempo, se produce un cambio de la velocidad de cada partícula. La aceleración de cada partícula se genera a partir de un término aleatorio. En los últimos años, la optimización por enjambre de partículas ha sido aplicada en muchas investigaciones y casos ingenieriles. Está demostrado que la optimización por enjambre de partículas obtiene mejores resultados de forma más rápida y más económica comparado con otros métodos [4].

En el algoritmo 1 se muestra el pseudocódigo de la operación del algoritmo PSO general [9].

En PSO, en cada iteración, cada $x_{i}$ se renueva dependiendo de dos valores, el óptimo local $x_{i}$, y el óptimo global $x$. la renovación se realiza según las ecuaciones 27 y 28.

$$
\begin{aligned}
& v_{i, j}(t+1)=w v_{i, j}(t)+c_{1} r_{1}\left[p_{i, j}-x_{i, j}(t)\right]+c_{2} r_{2}\left[p_{g, j}-x_{i, j}(t)\right], \\
& x_{i, j}(t+1)=x_{i, j}(t)+v_{i, j}(t+1), j=1,2, \ldots, \text { número de laminas. }
\end{aligned}
$$

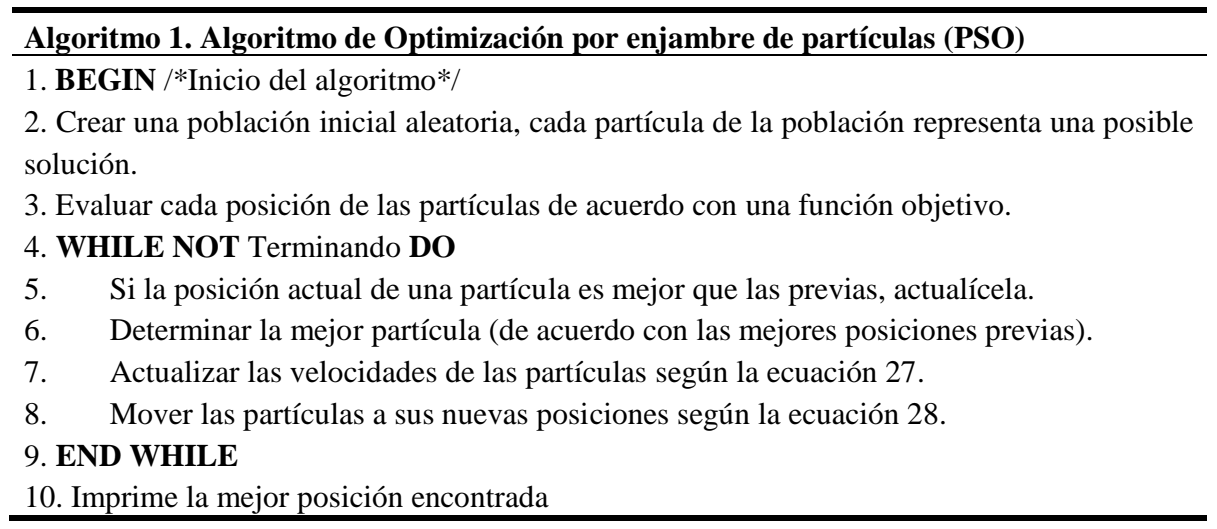

\subsection{Planteamiento del problema de optimización}

Diseñar un árbol de transmisión de materiales compuestos (figura 5), en donde las variables a optimizar son el número de láminas y la secuencia de apilamiento, suponiendo que el laminado es simétrico. La función objetivo establecida para este problema es el peso del árbol de transmisión, como se muestra en la ecuación 29. 




Fig. 5. Árbol de transmisión de materiales compuestos.

$$
\min f(x)=m=\rho \frac{\pi}{4}\left(d_{0}^{2}-d_{i}^{2}\right) L,
$$

vector de las variables de diseño $\quad x=\left[n, \theta_{k}, d_{i}\right]$.

Sujeto a las siguientes restricciones de diseño:

$\mathrm{n}>1, \quad$ donde $n \in$ números enteros

$-90 \leq \theta_{\mathrm{k}} \leq 90$

$\mathrm{Tcr} \geq \mathrm{Tmax}$

Ncr $\geq$ Nmax

Si se ha producido rotura $\rightarrow \mathrm{m}=\infty$
Donde:

$\rho=$ densidad del material

$n=$ número de láminas

$\theta_{k}=$ orientación de las fibras

$d_{i}=$ espesor del árbol

$d_{o}=$ diámetro exterior

$m=$ masa

$T c r=$ resistencia a deformación por torsión

Tmax $=$ par de servicio

$\mathrm{Ncr}=$ frecuencia natural

$N \max =$ velocidad de giro

\subsection{Fuerzas externas que actúan sobre el sistema}

Las fuerzas o cargas externas que sufre un árbol de transmisión con sección hueca, sometido al torque aplicado y considerando la fuerza centrífuga (véase figura 6), resultan como se describe en las ecuaciones 30,31 y 32 .

$$
\begin{aligned}
N x & =0, \\
N y & =2 t \rho r^{2} w^{2}, \\
N x y & =\frac{T}{2 \pi r} .
\end{aligned}
$$




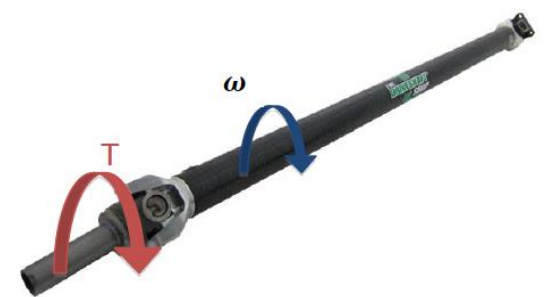

Fig. 6. Fuerzas externas que actúan sobre el árbol de transmisión.
Donde:

$\rho=$ densidad del material $\omega=$ velocidad angular $t=$ espesor de la pieza $r=$ radio de la sección $T=$ torque aplicado $N_{x}=$ carga axial

Se propone el material mostrado en la tabla 1 para realizar el diseño del árbol, se muestra las propiedades mecánicas del material compuesto de Carbono/Epoxi USN150. Las direcciones materiales de una lámina se muestran en la figura 7 [6].

Tabla 1. Material propuesto para el diseño del árbol de transmisión.

\begin{tabular}{|c|c|c|c|}
\hline Concepto & & Unidad & $\begin{array}{l}\text { Carbono/Epoxi } \\
\text { (USN150) }\end{array}$ \\
\hline Módulo de elasticidad en dirección 1 & $E_{1}$ & GPa & 131,6 \\
\hline Módulo de elasticidad en dirección 2 y 3 & $E_{2}, E_{3}$ & $\mathrm{GPa}$ & 8,20 \\
\hline Módulo de cortante en plano 23 & $G_{23}$ & GPa & 3,5 \\
\hline Módulo de cortante en plano 13 y 12 & $G_{13}, G_{12}$ & $\mathrm{GPa}$ & 4,5 \\
\hline Coef. de Poisson en plano 12 y 13 & $v_{12}, v_{13}$ & -- & 0,282 \\
\hline $\begin{array}{l}\text { Coef. de dilatación térmica en } \\
\text { dirección } 1\end{array}$ & $\alpha_{1}$ & $\times 10^{-6} /{ }^{\circ} \mathrm{C}$ & $-0,9$ \\
\hline $\begin{array}{l}\text { Coef. de dilatación térmica en } \\
\text { dirección } 2\end{array}$ & $\alpha_{2}$ & $\times 10^{-6} /{ }^{\circ} \mathrm{C}$ & 27 \\
\hline $\begin{array}{l}\text { Coef. de expansión higroscópico en } \\
\text { dirección } 1\end{array}$ & $\beta_{1}$ & -- & 0 \\
\hline $\begin{array}{l}\text { Coef. de expansión higroscópico en } \\
\text { dirección } 2\end{array}$ & $\beta_{2}$ & --- & 0,4 \\
\hline Resistencia a la tracción en dirección 1 & $S_{1}^{t}$ & MPa & 2000 \\
\hline $\begin{array}{l}\text { Resistencia a la compresión en } \\
\text { dirección } 1\end{array}$ & $S_{1}^{c}$ & MPa & 1400 \\
\hline $\begin{array}{l}\text { Resistencia a la tracción en } \\
\text { dirección } 2 \text { y } 3\end{array}$ & $S_{2}^{t}, S_{3}^{t}$ & MPa & 61 \\
\hline $\begin{array}{l}\text { Resistencia a la compresión en } \\
\text { dirección } 2 \text { y } 3\end{array}$ & $S_{2}^{c}, S_{3}^{c}$ & MPa & 130 \\
\hline Resistencia al cortante en plano 23 & $S_{23}$ & MPa & 40 \\
\hline Resistencia al cortante en plano 13 y 12 & $S_{13}, S_{12}$ & $\mathrm{MPa}$ & 70 \\
\hline Densidad & $\rho$ & $\mathrm{kg} / \mathrm{m}^{3}$ & 1550 \\
\hline Espesor de la lámina & $t_{\text {lámina }}$ & $\mathrm{mm}$ & 0,125 \\
\hline
\end{tabular}

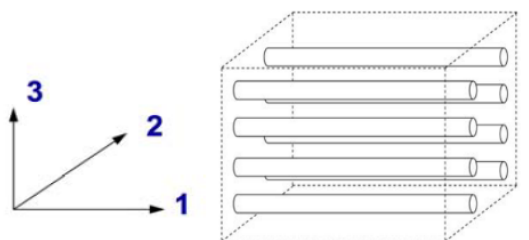

Fig. 7. Direcciones materiales en una lámina. 


\section{Implementación computacional}

La implementación del algoritmo se programó en el entorno de MATLAB $®$ R2015a, y las corridas se llevaron a cabo en una plataforma computacional con las siguientes características: procesador Intel Core i3 @ $1.80 \mathrm{GHz}$, con 12GB de memoria RAM y Sistema operativo Windows 10.

Los datos de entrada al algoritmo PSO se muestran en la tabla 2, en donde se consideran los parámetros de fabricación de la pieza, del algoritmo, geométricos y cargas externas.

Tabla 2. Datos de entrada al algoritmo PSO.

\begin{tabular}{|c|c|c|}
\hline \multicolumn{3}{|c|}{ Datos de entrada: } \\
\hline Velocidad máxima de giro & $9000 \mathrm{rpm}$ & \multirow{4}{*}{$\begin{array}{l}\text { Cargas externas } \\
\text { Parámetros geométricos }\end{array}$} \\
\hline Par máximo transmitido & $3500 \mathrm{Nm}$ & \\
\hline Diámetro exterior & $0.1 \mathrm{~m}$ & \\
\hline Longitud del árbol & $1.5 \mathrm{~m}$ & \\
\hline Temperatura de curado & $120^{\circ} \mathrm{C}$ & \multirow{4}{*}{ Parámetros de fabricación de la pieza } \\
\hline Temperatura de servicio & $20^{\circ} \mathrm{C}$ & \\
\hline Contenido de humedad & $0.5 \%$ & \\
\hline Coeficiente de seguridad & 2.5 & \\
\hline Tamaño de población & 50 & \multirow{2}{*}{ Parámetros del algoritmo } \\
\hline Número máximo de iteraciones & 100 & \\
\hline
\end{tabular}

Consideraciones basadas en la literatura para obtener el número óptimo de láminas mediante el uso del algoritmo PSO [4]:

- 10-32 (experimentalmente se determina que por debajo de 10 láminas es imposible tener un diseño factible, mientras que con 32 se puede garantizar la existencia del diseño factible).

- $\quad \mathrm{N}=$ tamaño de la población, para PSO normalmente es entre 40 y 100.

- $\quad \mathrm{w}=$ factor de inercia, típicamente 0.5 .

- $\quad \mathrm{c} 1 \mathrm{y} \mathrm{c} 2=$ factores de aprendizaje, normalmente se toma 2.

- $\quad \mathrm{r} 1$ y r2=son números aleatorios entre 0 y 1 .

\section{Resultados y discusión}

Estos resultados son valores promedio de haber realizado múltiples corridas del algoritmo y de una selección de muestras de estudio de 30 corridas, las cuales convergieron a la solución. La tabla 3 muestra un resumen de los parámetros de diseño obtenidos por el algoritmo para un árbol de transmisión hecho de fibra de carbono/epoxi USN150, mientras que en la figura 8 se observa la evolución de la búsqueda, mencionando que a cada iteración se aumenta una lámina para realizar una búsqueda exhaustiva, y de las soluciones factibles tomar la de menor valor de función objetivo. 
Tabla 3. Resultados del algoritmo PSO para el diseño de un árbol de transmisión hecho de fibra de carbono/epoxi.

\begin{tabular}{|c|c|c|c|c|c|c|c|c|c|c|c|c|c|}
\hline $\mathbf{i}$ & $\begin{array}{c}\text { do } \\
(\mathrm{mm})\end{array}$ & $\stackrel{\mathrm{L}}{(\mathrm{mm})}$ & $\begin{array}{c}\text { tk } \\
(\mathrm{mm})\end{array}$ & $\mathrm{n}$ & $\stackrel{t}{t}$ & $\begin{array}{c}\text { Secuencia } \\
\text { Óptima }\left(\theta_{k}\right)\end{array}$ & $\begin{array}{l}\operatorname{Tmax} \\
(\mathrm{Nm})\end{array}$ & $\begin{array}{l}\text { Nmax } \\
(\mathrm{rpm})\end{array}$ & $\underset{(\mathrm{kg})}{\mathrm{m}}$ & $\begin{array}{l}\text { Tc } \\
\text { curado } \\
\left({ }^{\circ} \mathrm{C}\right)\end{array}$ & $\begin{array}{c}\text { Ts } \\
\text { servicio } \\
\left({ }^{\circ} \mathrm{C}\right)\end{array}$ & $\begin{array}{c}\% \\
\text { humedad }\end{array}$ & cs \\
\hline 1 & 100 & 1500 & 0.125 & 22 & 2.75 & {$[18 / 84 / 13 /-51 / 9 / 62 /-50 /-30 / 38 /-7 / 62] \mathrm{s}$} & 3500 & 9000 & 1.953417 & 120 & 20 & 0.5 & 2.5 \\
\hline 2 & 100 & 1500 & 0.125 & 22 & 2.75 & {$[1 /-86 / 16 /-7 /-71 / 33 / 37 / 7 /-47 / 33 / 58] \mathrm{s}$} & 3500 & 9000 & 1.953417 & 120 & 20 & 0.5 & 2.5 \\
\hline 3 & 100 & 1500 & 0.125 & 22 & 2.75 & {$[-21 / 16 /-15 /-21 / 37 /-14 / 0 /-82 / 45 /-20 /-22] \mathrm{s}$} & 3500 & 9000 & 1.953417 & 120 & 20 & 0.5 & 2.5 \\
\hline 4 & 100 & 1500 & 0.125 & 22 & 2.75 & {$[-23 /-20 /-58 / 7 / 38 /-26 /-22 / 55 /-82 / 53 /-] 5$} & 3500 & 9000 & 1.953417 & 120 & 20 & 0.5 & 2.5 \\
\hline 5 & 100 & 1500 & 0.125 & 22 & 2.75 & {$[0 / 11 /-20 /-48 / 47 / 15 / 53 /-41 /-22 / 35] \mathrm{s}$} & 3500 & 9000 & 1.953417 & 120 & 20 & 0.5 & 2.5 \\
\hline 6 & 100 & 1500 & 0.125 & 22 & 2.75 & {$[1 /-29 / 50 /-47 /-35 / 74 / 20 / 8 /-57 /-11 /-70] \mathrm{s}$} & 3500 & 9000 & 1.953417 & 120 & 20 & 0.5 & 2.5 \\
\hline 7 & 100 & 1500 & 0.125 & 22 & 2.75 & {$[49 /-51 /-38 /-5 /-65 / 15 / 0 /-28 /-6 /-35 /-17] \mathrm{s}$} & 3500 & 9000 & 1.953417 & 120 & 20 & 0.5 & 2.5 \\
\hline 8 & 100 & 1500 & 0.125 & 22 & 2.75 & {$[-4 /-62 /-87 / 34 /-47 /-47 /-31 / 5 /-20 /-5 / 5] \mathrm{s}$} & 3500 & 9000 & 1.953417 & 120 & 20 & 0.5 & 2.5 \\
\hline 9 & 100 & 1500 & 0.125 & 22 & 2.75 & {$[-10 /-5 /-13 /-22 /-22 /-56 /-2 / 21 /-17 / 32 / 6]_{5}$} & 3500 & 9000 & 1.953417 & 120 & 20 & 0.5 & 2.5 \\
\hline 10 & 100 & 1500 & 0.125 & 22 & 2.75 & {$[14 / 46 /-27 / 82 / 18 /-13 /-6 / 76 / 47 /-27 /-36] \mathrm{s}$} & 3500 & 9000 & 1.953417 & 120 & 20 & 0.5 & 2.5 \\
\hline 11 & 100 & 1500 & 0.125 & 22 & 2.75 & {$[46 /-6 /-23 /-26 /-42 /-11 / 22 / 83 /-61 /-59 /-22] \mathrm{s}$} & 3500 & 9000 & 1.953417 & 120 & 20 & 0.5 & 2.5 \\
\hline 12 & 100 & 1500 & 0.125 & 22 & 2.75 & {$[49 /-27 / 20 /-4 / 41 / 14 / 46 /-5 / 43 /-69 / 14] \mathrm{s}$} & 3500 & 9000 & 1.953417 & 120 & 20 & 0.5 & 2.5 \\
\hline 13 & 100 & 1500 & 0.125 & 22 & 2.75 & {$[-76 / 30 /-14 /-51 / 87 / 35 /-21 / 35 /-41 /-49 / 34] \mathrm{s}$} & 3500 & 9000 & 1.953417 & 120 & 20 & 0.5 & 2.5 \\
\hline 14 & 100 & 1500 & 0.125 & 22 & 2.75 & {$[2 /-66 / 5 / 34 /-21 / 13 /-25 / 47 / 10 / 36 /-44] \mathrm{s}$} & 3500 & 9000 & 1.953417 & 120 & 20 & 0.5 & 2.5 \\
\hline
\end{tabular}

donde:

$d_{o}=$ diámetro exterior

$L=$ longitud

$t_{k}=$ espesor de una lámina

$n=$ número de láminas

$t=$ espesor total de la pieza

$\theta_{k}=$ orientación de las fibras
Tmax =par de servicio

Nmax =velocidad de giro

$m=$ masa

$T c=$ temperatura de curado de la pieza

$T s=$ temperatura de servicio

$\%=$ porcentaje de humedad

$C S=$ factor de seguridad en el diseño

\section{Carbono/epoxi: Peso vs iteración}

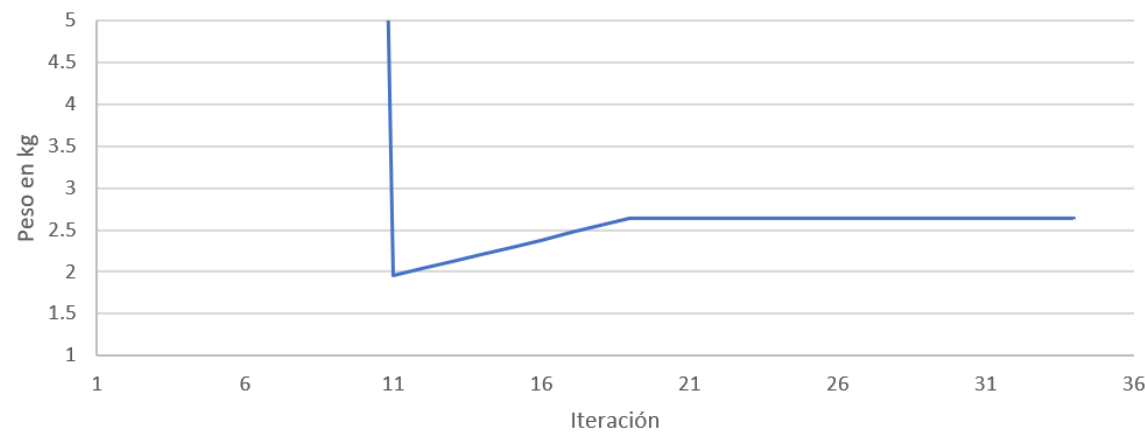

Fig. 8. Variación del peso del árbol de transmisión respecto al número de iteración.

Respecto a los resultados mostrados en la tabla 3, la nomenclatura de la orientación de las fibras o secuencia óptima de apilamiento es simétrica, es decir la $s$ al final del corchete significa que es la primera parte de los laminados respecto al eje medio y que la siguiente mitad tendrá las mismas orientaciones de forma simétrica. 
Además, se puede observar que mediante el algoritmo PSO se ha obtenido un diseño óptimo que tiene 22 láminas y un peso de $1.954 \mathrm{~kg}$, para todas las corridas que convergen al óptimo global, sin embargo, las secuencias de apilamiento cambian, esto es razonable ya que se trata de un algoritmo que crea soluciones aleatorias y las va mejorando a cada iteración, además de que existen demasiadas combinaciones posibles que llevan a obtener el objetivo buscado, que para este caso es el menor peso de material para soportar las cargas a las que estará sometida la pieza, por lo que se puede establecer que todas las secuencias de apilamiento son factibles, siempre y cuando converjan al mínimo peso y al número de láminas óptimo.

El costo computacional en este caso no es prioritario, ya que lo que interesa es el resultado, para converger este algoritmo aproximadamente tarda 8 minutos, mientras que en el trabajo presentado en [4] tarda 80 minutos, además la secuencia de apilamiento es muy teórica, por los valores obtenidos, ya que si se quiere fabricar la pieza es difícil obtener las orientaciones sugeridas, en la práctica se suele restringir el diseño a ángulos de $0^{\circ}, \pm 45^{\circ}$ y $90^{\circ}$ para facilitar la fabricación.

En trabajos previos, como en [4], para parámetros similares de diseño se han obtenido el mismo número de 22 láminas y un peso similar de $1.95 \mathrm{~kg}$, pero solo reportan una secuencia de apilamiento, sin embargo, ya se explicó que puede haber varias secuencias validas que cumplen con los parámetros establecidos. Mientras que en [5] utilizando parámetros similares, pero no iguales, por ejemplo, el material propuesto, y mediante algoritmos genéticos obtienen 17 láminas y un peso de $4.44 \mathrm{~kg}$. Estos resultados dan certidumbre a los obtenidos por la implementación propuesta en este trabajo.

\section{Conclusiones y trabajo futuro}

Se ha logrado implementar un algoritmo que es capaz de optimizar la secuencia de apilamiento y el número de láminas de fibra de carbono/epoxi para el diseño de un árbol de transmisión, el cual estará sometido a cargas especificas en su funcionamiento, de esta manera, el diseñador podrá ahorrar tiempo en el proceso de diseño cuando requiera de un análisis de este tipo de elementos, variando dentro del código del algoritmo los parámetros necesarios como, la longitud árbol de transmisión, la velocidad de servicio, el diámetro exterior, la temperatura de curado, la humedad del material, así como las características de otro material propuesto. Se puede establecer que la implementación de metaheurísticas en el diseño mediante materiales compuestos es de gran utilidad y con resultados favorables, debido a la complejidad de este tipo de diseños y al gran número de posibles soluciones existentes.

Con el fin de continuar con el desarrollo alcanzado por este trabajo, se propone implementar otros algoritmos evolutivos y de inteligencia colectiva para el diseño con materiales compuestos en diversas aplicaciones, como por ejemplo en el diseño de prótesis de fibra de carbono, con el objetivo de tener un parámetro de comparación entre diferentes metaheurísticas y así el diseñador pueda hacer uso de los resultados con base en la aplicación específica. 
Agradecimientos. Los autores agradecen al Instituto Politécnico Nacional, a la Sección de Estudios de Posgrado e Investigación de la Escuela Superior de Ingeniería Mecánica y Eléctrica Unidad Azcapotzalco, al Centro de Innovación y Desarrollo Tecnológico en Cómputo, al Tecnológico de Estudios Superiores de Ecatepec y al Consejo Nacional de Ciencia y Tecnología por el apoyo brindado.

\section{Referencias}

1. Suresh, S., Sujit, P. B., Rao, A. K.: Particle swarm optimization approach for multiobjetive composite box-beam desing. Composite Structures 81, pp. 598-605 (2007)

2. Vázquez Castillo, V., Hernández Lara, D., Merchán Cruz, E. A., Rodríguez Cañizo, R. G., Portilla Flores, E. A.: Implementación de algortimos genéticos para el diseño, optimización y selección de vigas. En: CORE 2017 (2017)

3. Reguera, F., Cortínez, V. H.: Diseño óptimo de vigas de pared delgada PRF sometidas a cargas dinámicas. Mecánica computacional, vol. XXXII, pp. 3575-3595 (2013)

4. Shengyu, W.: Uso de materiales compuestos en el diseño de un árbol de transmisión. España: Universidad Carlos III de Madrid (2014)

5. Rangaswamy, T., Vijayrangan, S.: Optimal sizing and stacking sequence of composite drive shafts. Materials science, vol. 11 No. 2. India (2005)

6. Navarro, C.: Elasticidad y resistencia de materiales II. España (2014)

7. Montaña, A. C. L. y J. L.: Algoritmos bioinspirados: la evolución biológica en la computación. Cantabria: Departamento de Matemáticas, Estadística y Computación, Universidad de Cantabria (2011)

8. Kennedy, J., Eberhart, R. C.: Intelligent Swarm Systems. New York, USA: Academic Press (2000)

9. Parsopoulos, K. E., Vrahatis, M. N.: Particle Swarm Optimization and Intelligence, Advances and Aplications. United States of America: Information Science Reference, (2010) 